Check for updates

Cite this: RSC Adv., 2019, 9, 36027

Received 25th September 2019

Accepted 31st October 2019

DOI: $10.1039 / c 9 r a 07793 f$

rsc.li/rsc-advances

\title{
Nanoceria-modified platinum supported on hierarchical zeolites for selective alcohol oxidation $\uparrow$
}

\author{
Marisa Ketkaew, ${ }^{a}$ Duangkamon Suttipat, (D) a Pinit Kidkhunthod, ${ }^{\text {b }}$ \\ Thongthai Witoon (D) ${ }^{c}$ and Chularat Wattanakit (D) *a
}

\begin{abstract}
The highly selective oxidation of alcohols to aldehydes has been achieved due to the synergic effect of Pt and $\mathrm{CeO}_{2}$ supported on hierarchical zeolites. The combination of $\mathrm{Pt}$ and $\mathrm{CeO}_{2}$ strongly enhances the catalytic performance of the oxidation of benzyl alcohol to benzaldehyde with respect to the isolated materials. In addition, the hierarchical zeolite not only increases the fraction of exposed active sites because of its high surface area that can prevent the aggregation of $\mathrm{Pt}$ and $\mathrm{CeO}_{2}$ nanoparticles, but also affects the oxidation state of cerium. The presence of a high content of trivalent $\mathrm{Ce}$ species $\left(\mathrm{Ce}^{3+}\right)$ on the hierarchical zeolite benefits the oxidation reaction, eventually leading to almost $100 \%$ yield of an aldehyde product. Moreover, the catalytic performance can be further improved by the easily tunable $\mathrm{Si}$ to Al ratio of zeolite catalysts.
\end{abstract}

\section{Introduction}

The selective oxidation of alcohols to aldehydes is of crucial importance to produce various intermediate compounds in fine chemicals. ${ }^{1}$ In particular, the oxidation of benzyl alcohol to benzaldehyde is one of the most fascinating processes because aromatic aldehydes can be used in various potential applications, for example as an industrial solvent, a commercial food flavouring, and an interesting key intermediate for perfumes and dyes. ${ }^{2}$ Typically, the synthesis of benzaldehyde has been achieved by the hydrolysis of benzylidene chloride or the partial oxidation of toluene. ${ }^{3}$ However, it often suffers from a low yield of product and a wastewater disposal problem. ${ }^{4}$ An alternative way to efficiently and cleanly produce benzaldehyde is the direct selective oxidation of liquid benzyl alcohol, which has been utilized under mild reaction conditions at a moderate reaction temperature. ${ }^{5}$

Indeed, various catalysts have been applied for the selective oxidation, in particular, $\mathrm{Cu}, \mathrm{Pd}, \mathrm{Au}$ and $\mathrm{Pt}^{6-8}$ for example. Although noble metals such as $\mathrm{Pt}, \mathrm{Pd}$ and $\mathrm{Au}^{9-11}$ play a crucial role as beneficial metal catalysts, there are still problems

\footnotetext{
${ }^{a}$ Department of Chemical and Biomolecular Engineering, School of Energy Science and Engineering, Nanocatalysts and Nanomaterials for Sustainable Energy and Environment Research Network NANOTEC, Vidyasirimedhi Institute of Science and Technology, Rayong 21210, Thailand. E-mail: Chularat.w@vistec.ac.th

${ }^{b}$ Synchrotron Light Research Institute (Public Organization), 111 University Avenue, Muang District, Nakhon Ratchasima, 30000 Thailand

${ }^{c}$ Faculty of Engineering, Department of Chemical Engineering, Kasetsart University, Bangkok 10900, Thailand

$\uparrow$ Electronic supplementary information (ESI) available: Details of experimental procedures and material characterization. See DOI: 10.1039/c9ra07793f
}

according to their cost effectiveness. Recently, several researchers have developed the catalysts to circumvent this limitation by decreasing the amount of noble metal via the combination with other materials. For instance, Evangelisti et $a .^{12}$ developed the hybrid bimetallic $\mathrm{Au} / \mathrm{CuO}$ core-shell nanoparticles to enhance the catalytic performance of alcohol oxidation due to the synergistic effect of $\mathrm{Au}$ and $\mathrm{CuO}$ nanostructures. Chen et al. ${ }^{13}$ also studied the synergistic effect of the bimetallic catalyst, which is $\mathrm{Au}-\mathrm{Pd}$ supported on ceria-zirconia. They revealed that the influence of the different Au-Pd interactions obtained by the different synthesis method directly related to the catalytic activity. In addition, the promising strategy to reduce the cost of catalysts and to sustainably improve the catalytic activity would be achieved by adding inexpensive metal oxide materials such as $\mathrm{CeO}_{2}, \mathrm{ZrO}_{2}$ and $\mathrm{TiO}_{2} \cdot{ }^{14,15}$ Among them, the $\mathrm{CeO}_{2}$ modified noble metal is one of the most interesting catalysts for the alcohol oxidation because of their outstanding characteristics such as the redox properties of $\mathrm{Ce}^{3+}$ and $\mathrm{Ce}^{4+}$ species, high oxygen vacancy capacity and poisoning tolerance of hydrocarbon on metal surfaces. ${ }^{16,17}$ The combination of them can greatly enhance the catalytic performance compared to isolated metals. In addition, it has been described that doping of ceria with silica or alumina can also transform ceria crystallites, eventually resulting in changing its oxidation states. ${ }^{18}$

As above-mentioned development of effective catalysts, other solid materials such as mesoporous silicas, ${ }^{19}$ molecular sieves,${ }^{20}$ montmorillonite ${ }^{21}$ and medicinal stone ${ }^{22}$ have been used to enhance the catalytic performance in many crucial oxidation processes. To date, many reports of the developed catalysts for selective oxidation of benzyl alcohol have been launched. It has 
been emphasized to modify the surface properties of the support in order to not only enhance the active sites dispersion but also facilitate in activating alcohol molecules. ${ }^{23,24}$ However, the factor that has an influence on the catalytic performance for an oxidation is not only an easy accessibility of reactant molecules into the active sites but also the surface properties such as acid/base property of the support surface..$^{25-27}$ Therefore, the tunability of solid support properties would make more benefit for the catalyst development.

It is well-known that a zeolite is also one type of aluminosilicate compounds and has been widely used as a solid support for metal oxides because of its unique properties, such as highsurface area, high metal ion exchange capacity, and high thermal/hydrothermal stability. ${ }^{28}$ However, the presence of sole micropores imposes the intracrystalline diffusion limitation in the zeolite framework, eventually leading to low utilization of active sites. Recently, there have been several reports on the development of hierarchical zeolites possessing at least two levels of porous structures, such as a microporous feature together with meso- or/and microporous networks, ${ }^{29,30}$ which have been used to improve the accessibility and molecular transportation of guest molecules into the zeolite framework. ${ }^{31}$

Although the hierarchical zeolite might be one of the most promising candidates as a solid support for nanometal oxides due to an increase in surface area, resulting in improving the dispersion of metal, the development of combined catalysts between the nanoceria-modified platinum and the hierarchical zeolite as a solid support has not yet been demonstrated even though there would be many advantages, in particular the beneficial effect of hierarchical structures on the improvement of redox active species.

In this context, we present the development of nanoceriamodified platinum supported on hierarchical zeolites for the selective oxidation of alcohols to aldehydes. The effect of alumina content in the hierarchical zeolite framework on the catalytic performance was also systematically investigated. Furthermore, the structural analysis of developed catalysts was completed by means of various techniques such as X-ray diffraction (XRD), scanning electron microscopy (SEM), transmission electron microscopy (TEM), $\mathrm{H}_{2}$ temperature programmed reduction $\left(\mathrm{H}_{2}\right.$-TPR) and X-ray absorption near edge structure (XANES) to gain insights into the mechanistic perspectives of redox species on different supports, which affect the catalytic activity of the selective oxidation of alcohols.

\section{Experimental}

\section{Materials}

All chemicals were of analytical grade and used as received without further purification. Aluminium isopropoxide (SigmaAldrich, $\geq 98.0 \%$ ), tetraethyl orthosilicate (TEOS, SigmaAldrich, $\geq 99.0 \%$ ), sodium hydroxide ( $\mathrm{NaOH}$, Carlo Erba, $\geq 98.0 \%$ ) were used as aluminium source, silica source and mineralizing agent, respectively. Tetrapropylammonium hydroxide (TPAOH, 1.0 $\mathrm{M}$ in $\mathrm{H}_{2} \mathrm{O}$ ) and tetrabutylphosphonium hydroxide (TBPOH, Sigma-Aldrich, $40 \%$ in $\mathrm{H}_{2} \mathrm{O}$ ) were used as structure directing agents (SDAs) for conventional zeolites and hierarchical zeolites, respectively. Cerium acetate (TCI, >98.0\%) and tetraamineplatinum(II) nitrate (Sigma-Aldrich, $\geq 50.0 \% \mathrm{Pt}$ basis) were used as precursor materials for cerium oxide and platinum active sites, respectively. To study catalytic activity, benzyl alcohol (Sigma-Aldrich, $\geq 99.0 \%$ ), toluene (Merck, $\geq 99.9 \%$ ) and decane (Sigma-Aldrich, $\geq 99.0 \%$ ) were used as reactant, solvent and internal standard, respectively.

\section{Catalyst preparation}

Synthesis of conventional silicalite-1 zeolite (Silicalite1CON). ${ }^{32}$ The conventional silicalite- 1 zeolite was synthesized with the molar composition of $10 \mathrm{SiO}_{2}$ : $1 \mathrm{TPAOH}: 1.03 \mathrm{NaOH}: 400 \mathrm{H}_{2} \mathrm{O}$. After mixing TEOS $(7 \mathrm{~g})$ and TPAOH (3.42 g) as a silica-SDA solution, the sodium hydroxide $(0.14 \mathrm{~g})$ mixed with DI-water was slowly added into the silicaSDA solution, and it was then stirred at room temperature for $2 \mathrm{~h}$. After the aging process, the obtained gel was transferred to the Teflon-lined hydrothermal synthesis reactor and heated to $180{ }^{\circ} \mathrm{C}$ for 3 days. After that, the prepared sample was washed with DI-water, filtered and dried at $110{ }^{\circ} \mathrm{C}$. Finally, the Silicalite1-CON zeolite was calcined at $650{ }^{\circ} \mathrm{C}$ for $8 \mathrm{~h}$ to remove SDA.

Synthesis of hierarchical silicalite-1 zeolite (Silicalite1HIE). ${ }^{32}$ The hierarchical silicalite-1 zeolite was synthesized with the molar composition of $60 \mathrm{SiO}_{2}$ : $18 \mathrm{TBPOH}: 0.75 \mathrm{NaOH}: 600 \mathrm{H}_{2} \mathrm{O}$. After mixing TEOS $(8.67 \mathrm{~g})$ and $\mathrm{TBPOH}(8.62 \mathrm{~g})$ as a silica-SDA solution, the sodium hydroxide $(0.02 \mathrm{~g})$ mixed with DI-water was slowly added into silica-SDA solution, and it was then stirred at room temperature for $12 \mathrm{~h}$. After the aging process, the synthesis gel was transferred to the Teflon-lined hydrothermal synthesis reactor and heated to $130{ }^{\circ} \mathrm{C}$ for 2 days. After that, the prepared sample was washed with DI-water, filtered and dried at $110^{\circ} \mathrm{C}$. Finally, the Silicalite1-HIE zeolite was calcined at $650^{\circ} \mathrm{C}$ for $8 \mathrm{~h}$ to remove SDA.

Synthesis of hierarchical ZSM-5 zeolite (ZSM5-HIE). ${ }^{32}$ The hierarchical ZSM-5 zeolite with different $\mathrm{Si} / \mathrm{Al}$ ratios (100 and 400) were synthesized with the molar composition of $60 \mathrm{SiO}_{2}: x \mathrm{Al}_{2} \mathrm{O}_{3}: 18 \mathrm{TBPOH}: 0.75 \mathrm{NaOH}: 600 \mathrm{H}_{2} \mathrm{O}$, where $x$ was 0.075 and 0.3 . Firstly, TEOS, aluminium isopropoxide were mixed as a silica-alumina solution. The second solution containing ТВPOH (8.62 g), sodium hydroxide (0.02 g) and DI-water was slowly added into a silica-alumina solution, and then it was stirred at room temperature for $12 \mathrm{~h}$. After the aging process, the obtained gel was transferred to the Teflon-lined hydrothermal synthesis reactor and heated to $130{ }^{\circ} \mathrm{C}$ for 2 days. After that, the prepared sample was washed with DI-water, filtered and dried at $110{ }^{\circ} \mathrm{C}$. Finally, the ZSM5-HIE zeolite was calcined at $650{ }^{\circ} \mathrm{C}$ for $8 \mathrm{~h}$ to remove SDA.

Preparation of $\mathrm{Pt}-\mathrm{CeO}_{2}$ supported on zeolites. The assynthesized zeolites were mixed with the desired amount of cerium acetate and DI-water at room temperature for $24 \mathrm{~h}$, and then the solvent was evaporated by rotary evaporator. Subsequently, the obtained sample was dried at $100{ }^{\circ} \mathrm{C}$ overnight. The prepared catalysts were calcined at $550{ }^{\circ} \mathrm{C}$ for $6 \mathrm{~h}$ and denoted as $y \mathrm{CeO}_{2}$-Silicalite1-HIE, $y \mathrm{CeO}_{2}$-ZSM5-HIE, and $y \mathrm{CeO}_{2}-$ 
Silicalite1-CON for the hierarchical silicalite-1, the hierarchical ZSM-5, and the conventional silicalite- 1 , respectively $(y=0,5$, 10, 20 and $30 \mathrm{wt} \%$ of $\mathrm{CeO}_{2}$ ). After that, the platinum loading process was proceeded in a similar procedure. The $y \mathrm{CeO}_{2}{ }^{-}$ Silicalite1-HIE, $y \mathrm{CeO}_{2}$-ZSM5-HIE, and $y \mathrm{CeO}_{2}$-Silicalite1-CON were stirred with aqueous platinum solution at room temperature for $24 \mathrm{~h}$ followed by the solvent removal and drying at $100^{\circ} \mathrm{C}$ overnight. Finally, the prepared catalysts were calcined at $550{ }^{\circ} \mathrm{C}$ for $6 \mathrm{~h}$ and denoted as $z \mathrm{Pt} / y \mathrm{CeO}_{2}$-Silicalite1-HIE, $z \mathrm{Pt} /$ $y \mathrm{CeO}_{2}$-ZSM5-HIE, $z \mathrm{Pt} / y \mathrm{CeO}_{2}$-Silicalite1-CON, for the hierarchical silicalite-1, the hierarchical ZSM5, and the conventional silicalite- 1 , respectively $(z=0,0.5,1$ and $2 \mathrm{wt} \%$ of Pt).

\section{Characterization}

The XRD patterns of the prepared catalysts were investigated using Bruker D8 ADVANCE instrument with $\mathrm{CuK}_{\alpha}$ radiation (40 $\mathrm{kV}, 40 \mathrm{~mA}$ ) in the $2 \theta$ range of $5^{\circ}$ to $60^{\circ}$ with the step size of $0.02^{\circ}$ and the scan rate of $1^{\circ} \mathrm{min}^{-1}$. To study the morphology of prepared catalysts, SEM and TEM images were obtained from JEOL JSM-7610F microscope and JEOL JEM-ARM200F microscope at $200 \mathrm{kV}$, respectively. The textural properties were determined by a $\mathrm{N}_{2}$ adsorption-desorption technique at $-196^{\circ} \mathrm{C}$ operated on a MicrotracBEL, BELSORP-max model and all the prepared samples were degassed at $350^{\circ} \mathrm{C}$ for $24 \mathrm{~h}$ before the measurement. The specific surface area (SBET), micropore surface area and pore volume were calculated by the BrunauerEmmett-Teller (BET) method and $t$-plot method, respectively. The elemental analysis was studied by using wavelengthdispersive X-ray fluorescence spectrometer (WDXRF) performed on Bruker S8 TIGER ECO instrument. The $\mathrm{H}_{2}$-TPR measurements were used to investigate the reducibility properties of the obtained catalysts. The measurement was conducted using a BELCAT II instrument equipped with thermal conductivity detectors (TCD). Typically, the samples (0.05 g) were pre-treated at $300{ }^{\circ} \mathrm{C}$ for 1 hour with the heating rate of $10^{\circ} \mathrm{C} \mathrm{min}{ }^{-1}$ under the flow of $\mathrm{Ar}$ and then cooled down to $50^{\circ} \mathrm{C}$. After that, the temperature was increased from $50{ }^{\circ} \mathrm{C}$ to $900^{\circ} \mathrm{C}$ with the heating rate of $5^{\circ} \mathrm{C} \mathrm{min}^{-1}$ in a flow of $2 \mathrm{v} / \mathrm{v} \%$ of $\mathrm{H}_{2}$ in Ar $\left(50 \mathrm{ml} \mathrm{min}^{-1}\right)$. To observe the metal dispersion, it was performed on a BELCAT II instrument equipped with thermal conductivity detector (TCD) using $\mathrm{H}_{2}$-pulse method. The catalysts were first treated in the air flow at $350{ }^{\circ} \mathrm{C}$ for $30 \mathrm{~min}$ followed by purging with $\mathrm{Ar}$ for $15 \mathrm{~min}$, and then pretreated in the flow of $\mathrm{H}_{2}$ at $350^{\circ} \mathrm{C}$ for 20 min followed by purging with $\mathrm{Ar}$ for 20 min to remove weakly adsorbed $\mathrm{H}_{2}$. Finally, the samples were cooled down to room temperature in the flow of $\mathrm{Ar}$, and a series of $\mathrm{H}_{2}$ pulses was injected until the amount of $\mathrm{H}_{2}$ pulses reached a steady state value. The amount of adsorbed $\mathrm{H}_{2}$ was measured and the metal dispersion (MD) and the average diameter of particles (assuming with spherical metal particles) were calculated by using the following equations:

$$
\mathrm{MD}(\%)=\frac{V_{\mathrm{m}} \times \mathrm{MW} \times 10^{4}}{W \% \times \mathrm{SF}}
$$

$$
D_{\mathrm{a}}(\mathrm{nm})=\frac{6 \times 10^{3}}{\mathrm{MS} \times \rho}
$$

where $V_{\mathrm{m}}$ is monolayer adsorbed volume $\left(\mathrm{mol} \mathrm{g}^{-1}\right)$, MW is metal atomic weight $\left(\mathrm{g} \mathrm{mol}^{-1}\right), W \%$ is metal percentage in sample, $\mathrm{SF}$ is stoichiometric factor (molecule of gas per metal atom), MS is metal surface area $\left(\mathrm{m}^{2} \mathrm{~g}^{-1}\right)$ and $\rho$ is metal density $\left(\mathrm{g} \mathrm{cm}^{-3}\right)$, respectively. ${ }^{33} \mathrm{Ce}_{3}$-edge XANES spectra were carefully characterized to study the oxidation state of $\mathrm{Ce}$ by using X-ray absorption near-edge structure in transmission mode at the BL5.2 station at Siam Photon Laboratory (Synchrotron Light Research Institute (Public Organization), SLRI), Thailand.

\section{Catalytic activity test}

The benzyl alcohol oxidation was carried out using a batch reactor. Firstly, the prepared catalysts were activated at $350{ }^{\circ} \mathrm{C}$ under the flow of $\mathrm{H}_{2}\left(5 \mathrm{ml} \mathrm{min}^{-1}\right)$ for $2 \mathrm{~h}$. After that, a $0.08 \mathrm{~g}$ of catalyst was transferred to two-neck round-bottom flask connected with a condenser column as a batch reactor. Then, a $6 \mathrm{ml}$ of toluene and decane were mixed together with the catalyst as a solvent and an internal standard, respectively. The reactor was sealed and heated to $80{ }^{\circ} \mathrm{C}$ under vigorous stirring. The reactant, which is a $50 \mu \mathrm{l}$ of benzyl alcohol was added into the reactor. The flow of air was introduced into the reactor under an atmospheric pressure using the balloon. The quantification of products was done by analysis using the internal calibration method. The reactant and products were sampled and analyzed by a gas chromatograph (GC, Agilent 7890B) equipped with a flame ionization detector (FID) and PoraBOND Q capillary column $(25 \mathrm{~m} \times 0.32 \mathrm{~mm})$. Subsequently, benzyl alcohol conversion ( $X_{\text {benzyl }}$ alcohol $)$ and benzaldehyde selectivity ( $S_{\text {benzaldehyde }}$ ) were calculated by the following equations:

$$
\begin{gathered}
X_{\text {benzyl alcohol }}(\%)=\frac{n_{0}-n_{\mathrm{i}}}{n_{0}} \times 100 \% \\
S_{\text {benzaldehyde }}(\%)=\frac{n_{\text {benzaldehyde }}}{n_{0}-n_{\mathrm{i}}} \times 100 \%
\end{gathered}
$$

where $n_{0}$ and $n_{\mathrm{i}}$ are mole of benzyl alcohol at initial and subsequent time, respectively. The mass balance was calculated for all experiments in the range of $97.59 \pm 10.04 \%$.

\section{Results and discussion}

To verify the effect of different contents of metals on the physicochemical properties of catalysts, the crystalline structures of nanoceria-modified platinum supported on hierarchical silicalite-1 (Silicalite1-HIE) with various metal loadings were examined by X-ray powder diffraction (XRD) as shown in Fig. S1 and S2. $\dagger$ As expected, when increasing the $\mathrm{CeO}_{2}$ and Pt loading the characteristic peaks at $2 \theta$ of $28,33,48$, and $56^{\circ}$ and $40^{\circ}$ corresponding to $\mathrm{CeO}_{2}$ and $\mathrm{Pt}$, respectively, ${ }^{34}$ were visibly observed. In strong contrast to this, the crystallinity of the silicalite-1 framework corresponding to the MFI topology significantly decreases as a function of $\mathrm{CeO}_{2}$ loading. Compared to other support materials, including the conventional silicalite- 
1 (Silicalite1-CON), hierarchical ZSM-5 (ZSM5-HIE) and commercial $\mathrm{CeO}_{2}$ nanoparticles, XRD patterns reveal that the nanoceria-modified platinum ( $1 \mathrm{wt} \%$ of $\mathrm{Pt}$ and $20 \mathrm{wt} \%$ of $\mathrm{CeO}_{2}$ ) supported on the conventional silicalite- $1\left(1 \mathrm{Pt} / 20 \mathrm{CeO}_{2}\right.$ Silicalite1-CON) consists of the characteristic peaks of $\mathrm{CeO}_{2}$, which are similar to the pattern of $1 \mathrm{Pt} / \mathrm{CeO}_{2}$, whereas the corresponding peaks disappear in cases of both hierarchical supports (1Pt/20CeO ${ }_{2}$-Silicalite1-HIE and $1 \mathrm{Pt} / 20 \mathrm{CeO}_{2}-\mathrm{ZSM} 5-$ HIE) (Fig. 1A). It is therefore reasonable to assume that small $\mathrm{CeO}_{2}$ nanoparticles can be somewhat formed on hierarchical zeolite surfaces. Therefore, it clearly demonstrates that the $1 \mathrm{Pt} /$ $20 \mathrm{CeO}_{2}$-Silicalite1-HIE and $1 \mathrm{Pt} / 20 \mathrm{CeO}_{2}$-ZSM5-HIE compose of well-dispersed $\mathrm{CeO}_{2}$ and $\mathrm{Pt}$ nanoparticles, whereas the large aggregated particles are preferably deposited on the outermost surfaces of Silicalite1-CON.

The morphologies of all the prepared catalysts are observed by scanning electron microscope (SEM) images as shown in Fig. $\mathrm{S} 3 \uparrow$ confirming that the structures are not destroyed after the metal-loading process. The large particle size of Silicalite1CON can be clearly observed, whereas hierarchical supports show a smaller particle formed from the self-assembled nanolayers with the size in the range of 150 to $250 \mathrm{~nm}$.

To reveal the morphologies of metal nanoparticles on supports, transmission electron microscopy (TEM) images of $1 \mathrm{wt} \%$ of $\mathrm{Pt}$ and $20 \mathrm{wt} \%$ of $\mathrm{CeO}_{2}$ loaded on different supports (conventional and hierarchical ones, which are $1 \mathrm{Pt} / 20 \mathrm{CeO}_{2}$ Silicalite1-CON and $1 \mathrm{Pt} / 20 \mathrm{CeO}_{2}$-Silicalite1-HIE, respectively) are shown in Fig. 1B and Fig. S4. $\dagger$ Obviously, the aggregation of
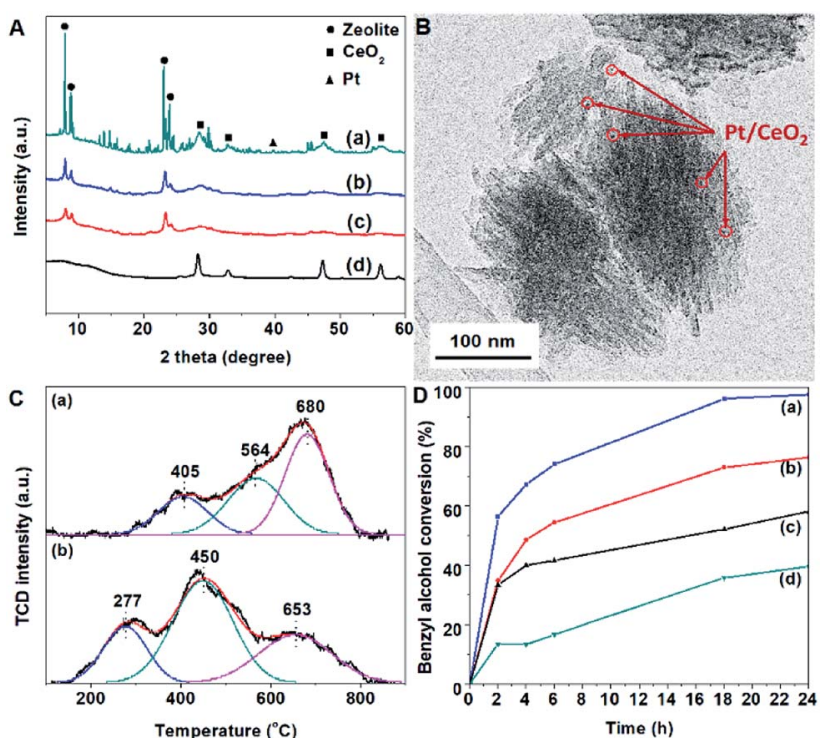

Fig. 1 Structural characterization of catalysts: (A) XRD patterns of nanoceria-modified platinum supported on different supports including (a) $1 \mathrm{Pt} / 20 \mathrm{CeO}_{2}$-Silicalite1-CON, (b) $1 \mathrm{Pt} / 20 \mathrm{CeO}_{2}$-Silicalite1$\mathrm{HIE}$, (c) $1 \mathrm{Pt} / 2 \mathrm{CCeO}_{2}-\mathrm{ZSM} 5-\mathrm{HIE}$, and (d) $\mathrm{Pt}-\mathrm{CeO}_{2}$; (B) TEM image of $1 \mathrm{Pt} / 20 \mathrm{CeO}_{2}$-Silicalite1-HIE; (C) $\mathrm{H}_{2}$-TPR profiles of (a) $1 \mathrm{Pt} / 20 \mathrm{CeO}_{2}-$ Silicalite1-CON and (b) $1 \mathrm{Pt} / 20 \mathrm{CeO}_{2}$-Silicalite1-HIE; (D) Catalytic activity of benzylalcohol oxidation over various catalysts: (a) 1Pt/ $20 \mathrm{CeO}_{2}-\mathrm{ZSM} 5-\mathrm{HIE}$, (b) $1 \mathrm{Pt} / 20 \mathrm{CeO}_{2}$-Silicalite1-HIE, (c) $1 \mathrm{Pt} / \mathrm{CeO}_{2}$ and (d) $1 \mathrm{Pt} / 20 \mathrm{CeO}_{2}$-Silicalite1-CON $(100 \%$ selectivity towards benzaldehyde). metals on the conventional samples is a main problem, whereas the metal distribution over Silicalite1-HIE is greatly improved. These behaviors are also confirmed by TEM-EDX (Fig. S5 $\dagger$ ) demonstrating a well-dispersed $\mathrm{Pt}$ and $\mathrm{CeO}_{2}$ over the hierarchical sample.

Furthermore, $\mathrm{N}_{2}$ physisorption isotherms are displayed in Fig. $66 \dagger$ and the textural properties are summarized in Table S1. $\dagger$ The conventional zeolite typically shows the type I isotherm, which can be observed for $1 \mathrm{Pt} / 20 \mathrm{CeO}_{2}$-Silicalite1CON, implying that it composes of a pure microporous structure. In contrast, the hierarchical zeolites exhibit mixed isotherms between types I and IV due to the micropore filling at the low relative pressure with a high uptake and a hysteresis loop at the high relative pressure attributed to a capillary condensation within mesopores and macropores, respectively. ${ }^{32}$ However, the isotherm of $1 \mathrm{Pt} / \mathrm{CeO}_{2}$ is totally different demonstrating that there is almost no adsorption ability at low relative pressure with the small hysteresis loop at high relative pressure, indicating non-porous structures with the interparticle void. ${ }^{35}$ These observations clearly show that hierarchical zeolites exhibit the higher specific surface area, which could provide the improved metal dispersion, eventually leading to enhance the catalytic performance. Although the $1 \mathrm{Pt} / \mathrm{CeO}_{2}$ composes of the smallest particle size obtained from SEM images (Fig. S3D †), it still suffers from the agglomeration of $\mathrm{CeO}_{2}$ nanoparticles, resulting in a very low surface area of metal oxides.

To clarify the metal dispersion ability, the $\mathrm{H}_{2}$ pulse chemisorption technique was also applied over nanoceria-modified platinum supported on different supports. The evaluation of the number of exposed active surfaces of Pt by chemisorption is based on the measurement of the amount of $\mathrm{H}_{2}$ gas that is adsorbed during the analytical cycle. In order to inhibit the hydrogen spillover, it has been recommended to chemisorb hydrogen at lower temperature. ${ }^{36}$ The adsorbed quantities are used to indicate the metal dispersion in accordance with the calculation. ${ }^{33}$ Interestingly, hierarchical zeolite supports can enhance the metal dispersion compared with the conventional zeolite (Table S1†). These observations again confirm the improved dispersion of $\mathrm{Pt}$ and $\mathrm{CeO}_{2}$ supported on hierarchical zeolites.

To investigate the redox properties of active sites, hydrogen temperature-programmed reduction $\left(\mathrm{H}_{2}-\mathrm{TPR}\right)$ profiles reveal that both $1 \mathrm{Pt} / 20 \mathrm{CeO}_{2}$-Silicalite1-CON and $1 \mathrm{Pt} / 20 \mathrm{CeO}_{2}$ Silicalite1-HIE compose of three predominant peaks at 200-350, 340-550 and 540-800 ${ }^{\circ} \mathrm{C}$ corresponding to the reduction of $\mathrm{PtO}_{x}$, the surface-active oxygen close to the $\mathrm{Pt}$-ceria interface and the bulk ceria, ${ }^{37}$ respectively (Fig. 1C). Notably, the reducibility of $\mathrm{PtO}_{x}$ at the $\mathrm{Pt}-\mathrm{CeO}_{2}$ interface plays an important role in an oxidation reaction. Interestingly, for $1 \mathrm{Pt} / 20 \mathrm{CeO}_{2}$-Silicalite1$\mathrm{HIE}, \mathrm{H}_{2}$ consumption was observed at lower temperature compared with that of $1 \mathrm{Pt} / 20 \mathrm{CeO}_{2}$-Silicalite1-CON, implying that Pt atoms were highly dispersed on the hierarchical support. These observations also agree with XRD and TEM results. It is therefore reasonable to assume that the hierarchical zeolite would promote highly efficient active sites to activate the interfacial lattice oxygen. These behaviors would increase the catalytic activity for the oxidation reaction. In addition, for 1Pt/ 
$20 \mathrm{CeO}_{2}$-ZSM5-HIE, the reducibility at $\mathrm{PtO}_{x}$ site is similar to what has been described in the case of $1 \mathrm{Pt} / 20 \mathrm{CeO}_{2}$-Silicalite1-HIE. Meanwhile, more distinct peaks can be observed in the sample of $1 \mathrm{Pt} / \mathrm{CeO}_{2}$ (Fig. $\mathrm{S} 7 \dagger$ ) due to the reduction of ceria surface and bulk ceria appeared at 400 and $800{ }^{\circ} \mathrm{C}$, respectively. ${ }^{38}$

To illustrate the beneficial effect of nanoceria-modified platinum and hierarchical zeolite on the selective oxidation of alcohols to aldehydes, the catalytic activities were tested using different Pt and $\mathrm{CeO}_{2}$ contents over the metal supported hierarchical silicalite-1 (Silicalite1-HIE) (Fig. 2). Notably, both isolated $\mathrm{Pt}$ and $\mathrm{CeO}_{2}$ exhibit a low catalytic activity for the oxidation of benzyl alcohol to benzaldehyde. However, the catalytic performance can greatly enhance when using a combined $\mathrm{Pt}$ and $\mathrm{CeO}_{2}$ catalyst, in particular, with $1 \mathrm{wt} \% \mathrm{of} \mathrm{Pt}$ and $20 \mathrm{wt} \%$ of $\mathrm{CeO}_{2}$ on hierarchical silicalite-1 as a solid support.

To study the effect of catalyst supports on the catalytic performance, as can be seen in Fig. 1D and 3, the catalytic activity of $1 \mathrm{Pt} / 20 \mathrm{CeO}_{2}$-Silicalite1-HIE is obviously improved compared with the $1 \mathrm{Pt} / 20 \mathrm{CeO}_{2}$-Silicalite1-CON. Interestingly, the highest yield of benzaldehyde can be achieved over 1Pt/ $20 \mathrm{CeO}_{2}$-ZSM5-HIE. In this case, ZSM5-HIE has the same framework as Silicalite1-HIE but it also consists of the Al sites in the framework, whereas the Silicalite1-HIE contains a pure silica (Table S1 $\dagger$ ). The reason for the higher catalytic activity of $1 \mathrm{Pt} / 20 \mathrm{CeO}_{2}$-ZSM5-HIE with respect to $1 \mathrm{Pt} / 20 \mathrm{CeO}_{2}$-Silicalite1HIE relates to the fact that the presence of alumina in the solid support can enhance the metal-support interaction. ${ }^{39}$ In addition, the catalytic performances also relate to the $\mathrm{Si} / \mathrm{Al}$ of hierarchical zeolites (Fig. 3). The low Si/Al provides the higher number of $\mathrm{Al}$ atoms incorporated into the framework, eventually resulting in the improvement of the catalytic activity. However, in the case of $1 \mathrm{Pt} / \mathrm{CeO}_{2}$, the catalytic performance is much lower than that of the hierarchical ones.

To understand the effect of active site structures on the catalytic activity, X-ray absorption near-edge spectroscopy (XANES) technique was used to investigate the oxidation state of

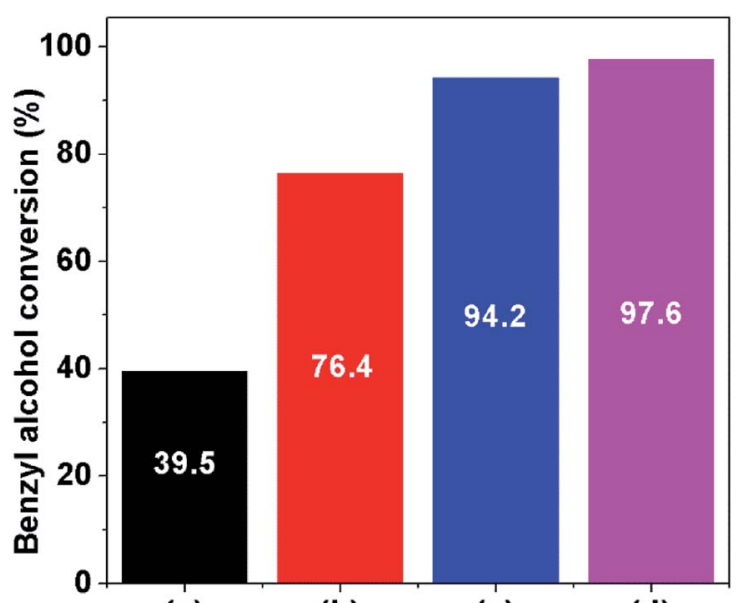

(a)

(b)

(c)

(d)

Fig. 3 Catalytic activity of the selective oxidation of benzyl alcohol to benzaldehyde (selectivity of benzaldehyde $=100 \%$ ) using a batch reactor at $80^{\circ} \mathrm{C}$ and 24 h over $1 \mathrm{wt} \% \mathrm{Pt}$ and $20 \mathrm{wt} \% \mathrm{CeO}_{2}$ supported on different support materials: (a) $1 \mathrm{Pt} / 20 \mathrm{CeO}_{2}-$ Silicalite1-CON; (b) $1 \mathrm{Pt} /$ $20 \mathrm{CeO}_{2}$-Silicalite1-HIE; (c) $1 \mathrm{Pt} / 20 \mathrm{CeO}_{2}-\mathrm{ZSM} 5(\mathrm{Si} / \mathrm{Al}=400$ )- $\mathrm{HIE}$; (d) $1 \mathrm{Pt} / 20 \mathrm{CeO}_{2}-\mathrm{ZSM} 5(\mathrm{Si} / \mathrm{Al}=100)-\mathrm{HIE}$.

Ce incorporated into the catalysts. Fig. 4 illustrates the

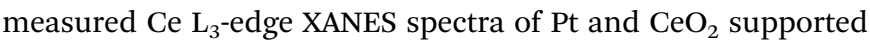
on different materials and two reference standards, $\mathrm{Ce}\left(\mathrm{NO}_{3}\right)_{3}$ $\cdot 6 \mathrm{H}_{2} \mathrm{O}$ and $\mathrm{CeO}_{2}$, which were used as $\mathrm{Ce}^{3+}$ and $\mathrm{Ce}^{4+}$ references, respectively. The peak $\mathrm{I}$ is associated to the absorption into the $5 \mathrm{~d}$ level with the $4 \mathrm{f}$ occupancy in the initial state, and therefore it indicates the presence of $\mathrm{Ce}^{3+}$ in the sample. While the double peaks (peaks II and III) relating to fully oxidized $\mathrm{CeO}_{2}$, are associated with the occupancy of the $4 \mathrm{f}$ level in the final state and the absorption into the $5 \mathrm{~d}$ level with no occupancy in the $4 \mathrm{f}$ level in either the initial or final state, respectively. Therefore, this peak is only expected when $\mathrm{Ce}^{4+}$ is present. ${ }^{40}$ The fitting of XANES spectra of the Ce $\mathrm{L}_{3}$ edge is demonstrated in Fig. S8. $\dagger$ The experimental curve can be reproduced by the combination of an arctan function in order to simulate the edge jump and fit
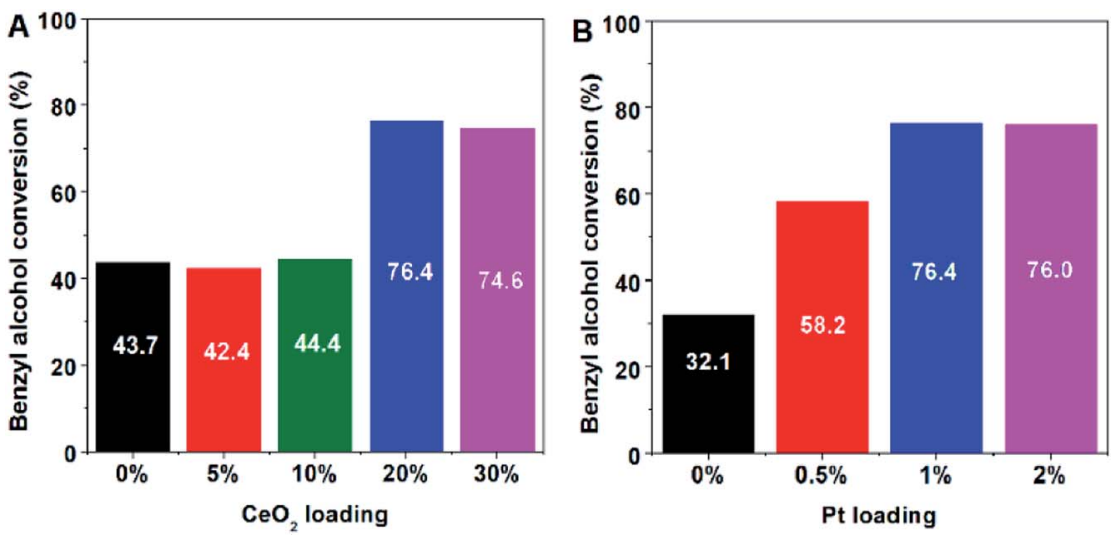

Fig. 2 Catalytic activity of the selective oxidation of benzyl alcohol to benzaldehyde (selectivity of benzaldehyde $=100 \%$ ) using a batch reactor at $80{ }^{\circ} \mathrm{C}$ and $24 \mathrm{~h}$ of reaction time over: (A) $1 \mathrm{wt} \%$ Pt supported on Silicalite1-HIE with different $\mathrm{CeO}_{2}$ loading; (B) 20 wt\% CeO 2 supported on Silicalite1-HIE with different Pt loading. 
I II III

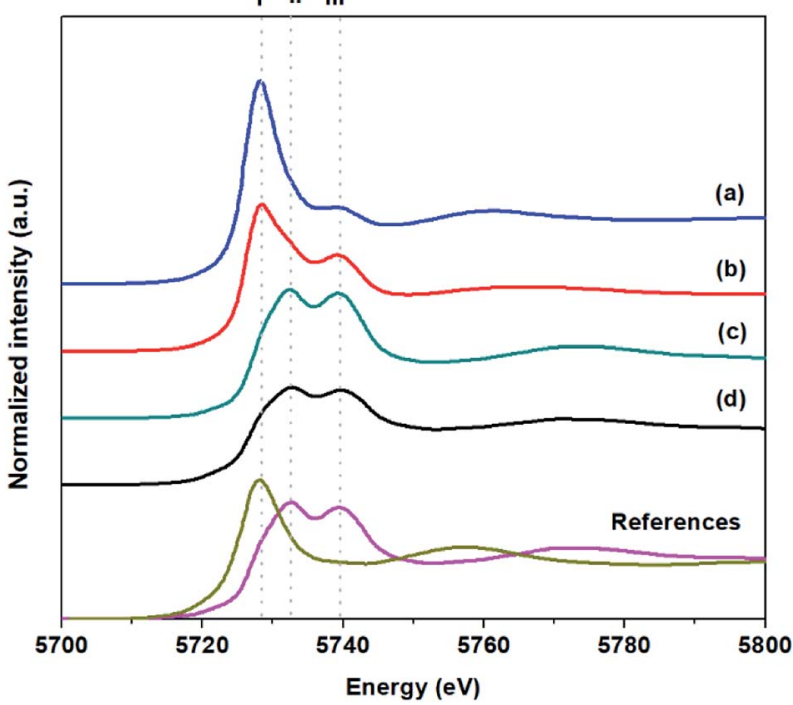

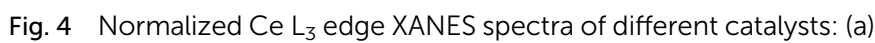
$1 \mathrm{Pt} / 20 \mathrm{CeO}_{2}-\mathrm{ZSM} 5-\mathrm{HIE}$, (b) $1 \mathrm{Pt} / 20 \mathrm{CeO}_{2}-$ Silicalite1-HIE, (c) $1 \mathrm{Pt} /$ $20 \mathrm{CeO}_{2}$-Silicalite1-CON, (d) $1 \mathrm{Pt} / \mathrm{CeO}_{2}$ and standard references of $\mathrm{Ce}^{3+}$ (yellow) and $\mathrm{Ce}^{4+}$ (pink).

three Gaussian functions (I, II, III). ${ }^{41}$ The quantitative analysis of Ce oxidation from XANES for prepared catalysts is summarized as shown in Table $\mathrm{S} 2 . \dagger$ In cases of $1 \mathrm{Pt} / 20 \mathrm{CeO}_{2}$-Silicalite1$\mathrm{CON}$ and $1 \mathrm{Pt} / \mathrm{CeO}_{2}$, their peaks can be absolutely fitted with $\mathrm{Ce}^{4+}$, whereas $1 \mathrm{Pt} / 20 \mathrm{CeO}_{2}$-Silicalite $1-\mathrm{HIE}$ and $1 \mathrm{Pt} / 20 \mathrm{CeO}_{2}{ }^{-}$ ZSM5-HIE exhibit a mixed phase between two valences with $\mathrm{Ce}^{3+}$ as a majority. The results of XANES indicate that support materials affect the oxidation state of Ce, resulting in the difference of catalytic performances. To clearly explain, $\mathrm{Ce}^{3+}$

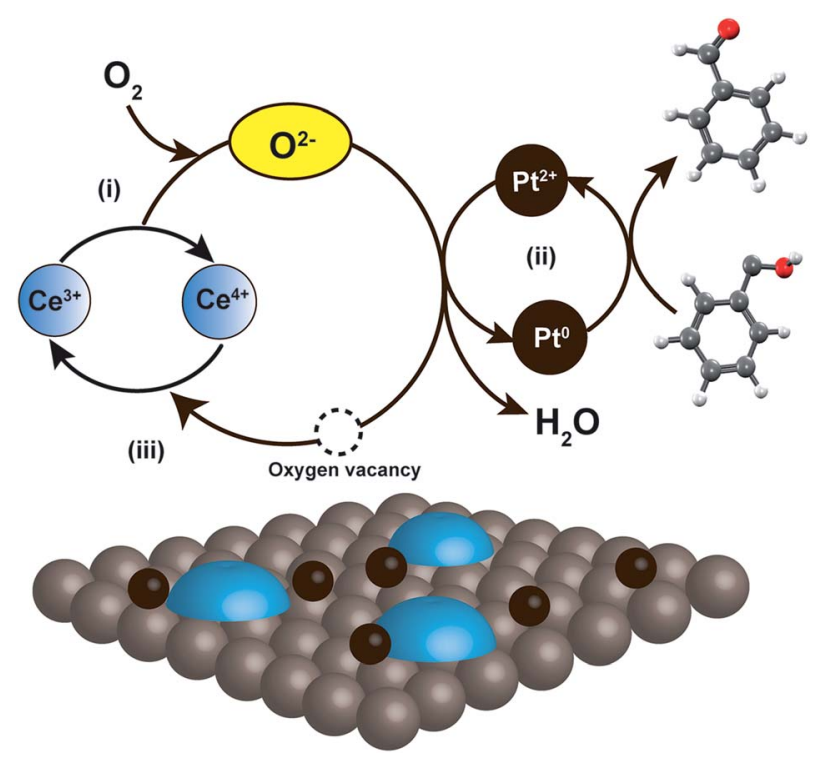

Fig. 5 Schematic illustration of proposed reaction mechanism obtained over nanoceria-modified platinum supported on hierarchical zeolites. usually comes from small particles of ceria nanoparticles, which is highly well-dispersed on solid supports. ${ }^{42}$ In cases of hierarchical catalysts, they show the higher catalytic activity compared to the conventional catalyst due to the oxygen vacancy of ceria, which can accelerate the mobility of lattice oxygen. However, in the case of $1 \mathrm{Pt} / \mathrm{CeO}_{2}$, the high $\mathrm{Ce}^{4+}$ content affects a lower catalytic performance compared with hierarchical supports. Interestingly, the oxidation state of Ce did not change after the reaction (Table $\mathrm{S} 2 \dagger$ ). This makes it clear that the active sites in this system were recyclable.

To propose the reaction mechanism over hierarchical catalysts, Fig. 5 shows the schematic illustration of the reaction mechanism including the following three main steps: (i) the reduced $\mathrm{Ce}^{3+}$ transferring an electron to $\mathrm{O}_{2}$, and producing $\mathrm{Ce}^{4+}$ and surface-active oxygen species $\left(\mathrm{O}^{2-}\right) ;{ }^{43}$ (ii) the formation of Pt-hydride by $\mathrm{H}$ atom adsorption from benzyl alcohol, which reacts with $\mathrm{O}^{2-}$ to produce $\mathrm{H}_{2} \mathrm{O}$ and an $\mathrm{O}$ vacancy; (iii) an $\mathrm{O}$ vacancy transferring to $\mathrm{Ce}^{4+}$, recovering reactive $\mathrm{Pt}^{0}$ and $\mathrm{Ce}^{3+}$ to produce fresh active sites for next catalytic cycles. ${ }^{44}$ Therefore, these features can be described as an electron bridge that facilitates the electron transfer from reductants to oxidants in the catalytic oxidation process.

\section{Conclusions}

The development of hierarchical zeolite catalysts was studied for the selective oxidation of benzyl alcohol to benzaldehyde as a model reaction. The synergic effect of $\mathrm{Pt}$ and $\mathrm{CeO}_{2}$ can greatly enhance the catalytic performance. Compared with a conventional zeolite, hierarchical zeolites strongly affect the catalytic activity because of not only improving the metal dispersion but also increasing $\mathrm{Ce}^{3+}$ fraction. Moreover, almost $100 \%$ yield of benzaldehyde can be achieved over $1 \mathrm{wt} \%$ of Pt combined with $20 \mathrm{wt} \%$ of $\mathrm{CeO}_{2}$ supported on hierarchical ZSM-5 having a low $\mathrm{Si} / \mathrm{Al}$ ratio. This example demonstrates the development of highly efficient catalyst with reducing the amount of noblemetal, which relates to environmental concerns and cost effectiveness, eventually leading to a potentially improved method for sustainable production of fine-chemicals.

\section{Conflicts of interest}

There are no conflicts to declare.

\section{Acknowledgements}

This work was supported by the Vidyasirimedhi Institute of Science and Technology, TTSF research project supported by Thailand Toray Science Foundation, Thailand Research Fund (MRG6180099), and the Office of Higher Education Commission. In addition, this work has been partially supported by the National Nanotechnology Center (NANOTEC), NSTDA, Ministry of Science and Technology, Thailand, through its program of Research Network NANOTEC. 


\section{Notes and references}

1 S. E. Davis, M. S. Ide and R. J. Davis, Green Chem., 2013, 15, 17-45.

2 R. Marotta, I. Di Somma, D. Spasiano, R. Andreozzi and V. Caprio, Chem. Eng. J., 2011, 172, 243-249.

3 J. A. Satrio and L. Doraiswamy, Chem. Eng. J., 2001, 82, 43-56.

4 C. Parmeggiani and F. Cardona, Green Chem., 2012, 14, 547564.

5 K. R. Seddon and A. Stark, Green Chem., 2002, 4, 119-123.

6 P. Gamez, I. W. Arends, J. Reedijk and R. A. Sheldon, Chem. Commun., 2003, 2414-2415.

7 B. Karimi, S. Abedi, J. H. Clark and V. Budarin, Angew. Chem., Int. Ed., 2006, 45, 4776-4779.

8 V. R. Choudhary, A. Dhar, P. Jana, R. Jha and B. S. Uphade, Green Chem., 2005, 7, 768-770.

9 H. Tsunoyama, H. Sakurai, Y. Negishi and T. Tsukuda, J. Am. Chem. Soc., 2005, 127, 9374-9375.

10 P. Maity, C. S. Gopinath, S. Bhaduri and G. K. Lahiri, Green Chem., 2009, 11, 554-561.

11 Q. Wang, X. Cai, Y. Liu, J. Xie, Y. Zhou and J. Wang, Appl. Catal., B, 2016, 189, 242-251.

12 M. Marelli, A. Jouve, A. Villa, R. Psaro, A. Balerna, L. Prati and C. Evangelisti, J. Phys. Chem. C, 2019, 123, 2864-2871.

13 C. M. Olmos, L. E. Chinchilla, A. Villa, J. J. Delgado, A. B. Hungría, G. Blanco, L. Prati, J. J. Calvino and X. Chen, J. Catal., 2019, 375, 44-55.

14 D. I. Enache, J. K. Edwards, P. Landon, B. Solsona-Espriu, A. F. Carley, A. A. Herzing, M. Watanabe, C. J. Kiely, D. W. Knight and G. J. Hutchings, Science, 2006, 311, 362365.

15 P. S. Murphin Kumar, S. Thiripuranthagan, T. Imai, G. Kumar, A. Pugazhendhi, S. R. Vijayan, R. Esparza, H. Abe and S. K. Krishnan, ACS Sustainable Chem. Eng., 2017, 5, 11290-11299.

16 A. Tanaka, K. Hashimoto and H. Kominami, J. Am. Chem. Soc., 2012, 134, 14526-14533.

17 C. Xu and P. K. Shen, Chem. Commun., 2004, 2238-2239.

18 E. Rocchini, A. Trovarelli, J. Llorca, G. W. Graham, W. H. Weber, M. Maciejewski and A. Baiker, J. Catal., 2000, 194, 461-478.

19 S. Wei, H. He, Y. Cheng, C. Yang, G. Zeng, L. Kang, H. Qian and C. Zhu, Fuel, 2017, 200, 11-21.

20 C. Yang, K. Zhao, Y. Cheng, G. Zeng, M. Zhang, J. Shao and L. Lu, Sep. Purif. Technol., 2016, 163, 153-161.

21 L. Kang, H. Liu, H. He and C. Yang, Fuel, 2018, 234, 12291237.

22 L. Qiu, Y. Cheng, C. Yang, G. Zeng, Z. Long, S. Wei, K. Zhao and L. Luo, RSC Adv., 2016, 6, 17036-17045.
23 P. Tamizhdurai, S. Sakthinathan, P. S. Krishnan, A. Ramesh, A. Abilarasu, V. L. Mangesh, S. Narayanan, K. Shanthi and T.-W. Chiu, Polyhedron, 2018, 155, 390-397.

24 J. Liu, Q. Yuan, H. Zhao and S. Zou, Catal. Lett., 2018, 148, 1093-1099.

25 S. S. Moeini, C. Battocchio, S. Casciardi, I. Luisetto, P. Lupattelli, D. Tofani and S. Tuti, Catalysts, 2019, 9, 847.

26 J. Xu, J.-K. Shang, Y. Chen, Y. Wang and Y.-X. Li, Appl. Catal., A, 2017, 542, 380-388.

27 C. S. Ramirez-Barria, M. Isaacs, C. Parlett, K. Wilson, A. Guerrero-Ruiz and I. Rodríguez-Ramos, Catal. Today, 2019, DOI: 10.1016/j.cattod.2019.05.057.

28 A. Corma, J. Catal., 2003, 216, 298-312.

29 L. Meng, B. Mezari, M. G. Goesten and E. J. M. Hensen, Chem. Mater., 2017, 29, 4091-4096.

30 L. Meng, X. Zhu, W. Wannapakdee, R. Pestman, M. G. Goesten, L. Gao, A. J. F. van Hoof and E. J. M. Hensen, J. Catal., 2018, 361, 135-142.

31 J. Pérez-Ramírez, C. H. Christensen, K. Egeblad, C. H. Christensen and J. C. Groen, Chem. Soc. Rev., 2008, 37, 2530-2542.

32 W. Wannapakdee, C. Wattanakit, V. Paluka, T. Yutthalekha and J. Limtrakul, RSC Adv., 2016, 6, 2875-2881.

33 M. Fadoni, L. Lucarelli and A. Dąbrowski, Stud. Surf. Sci. Catal., 1999, 120, 177-225.

34 X. Wang, D. Liu, S. Song and H. Zhang, J. Am. Chem. Soc., 2013, 135, 15864-15872.

35 F. J. Sotomayor, K. A. Cychosz and M. Thommes, Acc. Mater. Surf. Res., 2018, 3, 34-50.

36 V. Perrichon, L. Retailleau, P. Bazin, M. Daturi and J. C. Lavalley, Appl. Catal., A, 2004, 260, 1-8.

37 J. Ke, W. Zhu, Y. Jiang, R. Si, Y.-J. Wang, S.-C. Li, C. Jin, H. Liu, W.-G. Song and C.-H. Yan, ACS Catal., 2015, 5, 5164-5173.

38 R. Singha, A. Shukla, A. Yadav, T. Sasaki, A. Sandupatla, G. Deo and R. Bal, Catal. Sci. Technol., 2017, 7, 4720-4735.

39 J. Chen, Q. Zhang, Y. Wang and H. Wan, Adv. Synth. Catal., 2008, 350, 453-464.

40 S. Overbury, D. Huntley, D. Mullins and G. Glavee, Catal. Lett., 1998, 51, 133-138.

41 S. Phokha, S. Pinitsoontorn and S. Maensiri, Nano-Micro Lett., 2013, 5, 223.

42 C. M. Sims, R. A. Maier, A. C. Johnston-Peck, J. M. Gorham, V. A. Hackley and B. C. Nelson, Nanotechnology, 2018, 30, 085703.

43 Y. Chen, J. Chen, W. Qu, C. George, M. Aouine, P. Vernoux and X. Tang, Chem. Commun., 2018, 54, 10140-10143.

44 Z. Guo, B. Liu, Q. Zhang, W. Deng, Y. Wang and Y. Yang, Chem. Soc. Rev., 2014, 43, 3480-3524. 